\title{
Challenges in management of Severe Hemophilia with Acute Hematologic Malignancies
}

\author{
Mary Meyer ${ }^{1}$ and Akshat Jain ${ }^{2}$ \\ ${ }^{1}$ Loma Linda University \\ ${ }^{2}$ Loma Linda University Medical Center
}

May 18, 2020

\begin{abstract}
Approximately 13 patients with hemophilia A or B have been reported to be diagnosed with acute leukemia in childhood [1, 2]. This rarity appeared again in a 13 year old male with severe hemophilia with inhibitors who presented with worsening bone pain, weight loss, and leukocytosis. Coexistence of these life-threatening disorders, hemophilia and acute leukemia, pose practical challenges in providing standard care as the former has a propensity of bleeding and the latter requires intensive treatment with systemic and intrathecal chemotherapy. Our discussion aims at highlighting critical care strategies in management of severe hemophilia and hematologic malignancies.
\end{abstract}

\section{Discussion:}

We will discuss practical scenarios that may arise in Hemophilia management while treating cancer, including medications which result in thrombocytopenia, procedures with bleeding risk, possible development of Factor VIII inhibitor, and viral hepatitis and HIV infections, especially if the patient has been treated with plasma derived factor.

Chemotherapy, as used in cancer treatment, may cause thrombocytopenia and thus increases the risk of bleeding in a patient population which already has increased bleeding risk. Different drugs result in low platelet counts through different mechanisms, including affecting the stem cells (alkylating agents), the megakaryocyte progenitors (cyclophosphamide), or stopping megakaryocytes from releasing platelets (bortezomib) ${ }^{[5]}$. Frequent measurements of platelets are necessary throughout treatment to ensure the platelet threshold is kept and transfusion given if needed. Be sure to rule out any other reasons for thrombocytopenia besides the chemotherapy such as infection, worsening disease/metastasis, and heparin-induced thrombocytopenia [5].

For treatment of hemophilia, continuous bleeding prophylaxis is needed ${ }^{[6]}$. Doses may be administered 2-3 times a week depending on the type of hemophilia and other factors including venous access and the availability of clotting factors. With cancer, procedures which increase risk of a hematoma are often necessary. Specifically, lumbar punctures, bone marrow biopsies, and CVAD (port) or broviac catheter placement may be necessary with acute leukemia. During these procedures, one needs to prevent the development of a hematoma, and for the port and catheter placement, ensure clot formation to hold the line in place. To prevent a massive bleed, screen for inhibitors and have adequate amounts of clotting factor concentrates or plasma components available before the procedure ${ }^{[6]}$ and provide more of the appropriate factor prior to and after the procedure (Table 1). With this increased amount of factors, inhibitors may arise, so it is important to re-screen for inhibitors 4-12 weeks after the procedure ${ }^{[6]}$.

The inhibitors are IgG antibodies which neutralize clotting factors. You may suspect development of inhibitors if the expected recovery and half-life of factors are diminished. Of note, inhibitors are less likely to 
arise in hemophilia B than hemophilia ${ }^{[6]}$. When inhibitors arise, there are multiple options for treatment including high-dose clotting factor concentrates (increasing the amount of factor given or increasing the frequency of doses), bypassing agents (such as factor VII to provide an alternative method to complete the clotting cascade), products that mimic Factor VIII, and ITI Therapy (therapy which can clear the body of the inhibitors but takes weeks to months) ${ }^{[7]}$. Choosing a treatment method depends on multiple factors. For example, ITI therapy will not work fast enough for a procedure the next day, so you may consider a bypassing agent such as recombinant factor VIIa (rFVIIa) or activated prothrombin complex concentrates $(\mathrm{aPCC}){ }^{[6,8]}$.

It is also important to ensure patients with hemophilia are receiving safe factor products and screened for HIV or viral hepatitis. In the 1960s, fresh frozen plasma was used to stop bleeding episodes but sometimes resulted in patients being infected with HIV and viral hepatitis. Fortunately, improvements have occurred and since the 1990s coagulation factors products have not transmitted any clinically relevant pathogens ${ }^{[9]}$. In addition to inactivating viruses in the plasma, treatment further prevents infection with recombinant factor VIII and IX which can be used as well as bypassing agents. One should still be aware of the possibility of someone with hemophilia having HIV or viral hepatitis if they ever received plasma without viral inactivation, especially with cancer treatment which causes the patient to be immunocompromised.

\section{Conclusion:}

The concurrence of hemophilia and childhood leukemia is a rare occurrence. The patient described is the 14th severe hemophilia case reported in literature diagnosed with childhood leukemia and sets the precedent for management of complicated comorbidities in patients with severe Hemophilia. When treating patients with hemophilia for leukemia, one needs to consider the increased risks of breakthrough bleeding during chemotherapy. Items to be established at the onset include a hemophilia care plan listing factor VIII regimen tailored around chemotherapy and spinal tap, bypassing agents (recombinant VIIa $50-90 \mathrm{mcg} / \mathrm{kg}$ IV bolus q2hr until hemostasis achieved) for refractory breakthrough bleeding, and supportive care with antifibrinolytic agents (aminocaproic acid orally at $100 \mathrm{mg} / \mathrm{kg}$ every 6 hours, maximum 3 grams per dose) for minor mucosal bleeds. A target Factor VIII activity of $80-100 \%$ is recommended during invasive procedures. Due to likely development of thrombocytopenia with high dose chemotherapy, keeping platelet counts above 50 thousand $/ \mathrm{mm}^{3}$ is strongly recommended. Lastly surveillance for infections, especially HIV and viral hepatitis, must be performed prior to starting immunosuppressive chemotherapy.

Disclosures: No conflicts of interest to disclose

\section{References:}

1. Sinha, Rahul et al. A rare case of acute lymphoblastic leukaemia with hemophilia A. Italian Journal of Pediatrics December 2009. 35:40

2. A.L Dunn. Malignancy in patients with haemophilia: a review of the literature. Haemophilia 2010 . $16,427-436$

3. CDC. Hemophilia. Page last reviewed: May 6,2020.https://www.cdc.gov/ncbddd/hemophilia/data.html.

4. CDC: Morbidity and Mortality Weekly Report. Geographic Variation in Pediatric Cancer Incidence - United States, 2003-2014.Weekly / June 29, 2018 / 67(25);707713.https://www.cdc.gov/mmwr/volumes/67/wr/mm6725a2.htm

5. Kuter, D.J., Managing Thrombocytopenia Associated With Cancer Chemotherapy. Oncology Journal, Cancer Complications. Apr 15, 2015 29(4).

6. Srivastava et al. Guidelines for the management of hemophilia.Haemophilia . 2013. 19 (e1-e47)

7. CDC: Hemophilia: hemophilia and inhibitors. Page last reviewed: June 3, 2019.https://www.cdc.gov/ncbddd/hemophilia/inhibitors.html

8. Teitel, J.M. Treatment and prevention of bleeding in congenital hemophilia A patients with inhibitors. Transfusion and Apheresis Science. 2018. 57. 466-471

9. Mannucci, P.M. and Iacobelli, M. Progress in the contemporary management of hemophilia A: The new issue of patient aging. European Journal of Internal Medicine. 2017. 43. 16-21. 


\section{Hosted file}

Table 1.docx available at https://authorea.com/users/323150/articles/451988-challenges-inmanagement-of-severe-hemophilia-with-acute-hematologic-malignancies

\section{Hosted file}

Table 2.docx available at https://authorea.com/users/323150/articles/451988-challenges-inmanagement-of-severe-hemophilia-with-acute-hematologic-malignancies 